\title{
Immunology of Celiac Disease by Focused on Vaccine Therapy
}

\author{
Mohammad Rostami Nejad* \\ Celiac disease department, Gastroenterology and Liver diseases Research Center, Shahid Beheshti University of Medical Sciences, \\ Tehran, Iran \\ *Corresponding author:m.rostamii@gmail.com
}

Received September 07, 2015; Revised September 26, 2015; Accepted September 28, 2015

Cite This Article: Mohammad Rostami Nejad, "Immunology of Celiac Disease by Focused on Vaccine Therapy.” International Journal of Celiac Disease, vol. 3, no. 4 (2015): 115-117. doi: 10.12691/ijcd-3-4-4.

\section{Introduction}

Celiac disease (CD) is categorized by a strong genetic association with the MHC-II haplotype and about 95\% of patients carrying HLA-DQ2 haplotypes, and the remaining 5\% carrying HLA-DQ8 [1]. This relationship points to the principal role of the adaptive immunity of mucosal in the pathogenesis of CD.

This disorder is classically mediated by $\mathrm{T}_{\mathrm{H}} 1$ because of the enhanced mucosal mRNA expression of interferon (IFN)-gamma in untreated patients with CD [2]. Evidences have shown that $\mathrm{T}_{\mathrm{H}} 17 \mathrm{~T}$ cell subset (interleukin [IL]-17) are the main cells involved in the pathogenesis of autoimmune disorders and therefore it has become essential to consider the potential participation of $\mathrm{T}_{\mathrm{H}} 17$ cells in CD [2]. The result of different studies showed that the level of IL-17A is higher in patients with active CD in compare to patients on a gluten free diet (GFD). In addition, it has been confirmed that gliadin can induce the production of IL-1beta and IL-23 by $\mathrm{T}_{\mathrm{H}} 17$-polarizing in peripheral blood monocytes, and this connection providing a possible causative link between gluten exposure and $\mathrm{T}_{\mathrm{H}} 17$ cell expansion in CD [3].

On the other hands, it was shown that increased levels of adaptive immune markers such as IFN-gamma, IL-17A, IL-6 and IL-21 in the small intestinal mucosa of CD patients, are triggered by DQ2 DQ8 bounded tTG-deamidated gluten peptides and associated with $\mathrm{T}_{\mathrm{H}} 1$ - and $\mathrm{T}_{\mathrm{H}} 17-$ associated cytokine production and/or clone activation [4-9].

Among innate immune mechanisms, Toll-like receptors (TLRs) expression and function are also important in CD pathogenesis. These receptors class are able to detect microbial invasion and mediate a rapid inflammatory response. Among them TLR4 expression levels are higher on duodenal biopsies in CD cases compared to controls. Also high levels of IL-1, IL-6, IL-8, and IL-17 were detected in CD patients with high TLR4 levels $[10,11]$. On the other hands Kalliom"aki et al. reported that, TLR2 mRNA expression was significantly lower in untreated and treated celiac patients, while TLR9 mRNA expression was higher only in untreated subjects compared to controls [12].

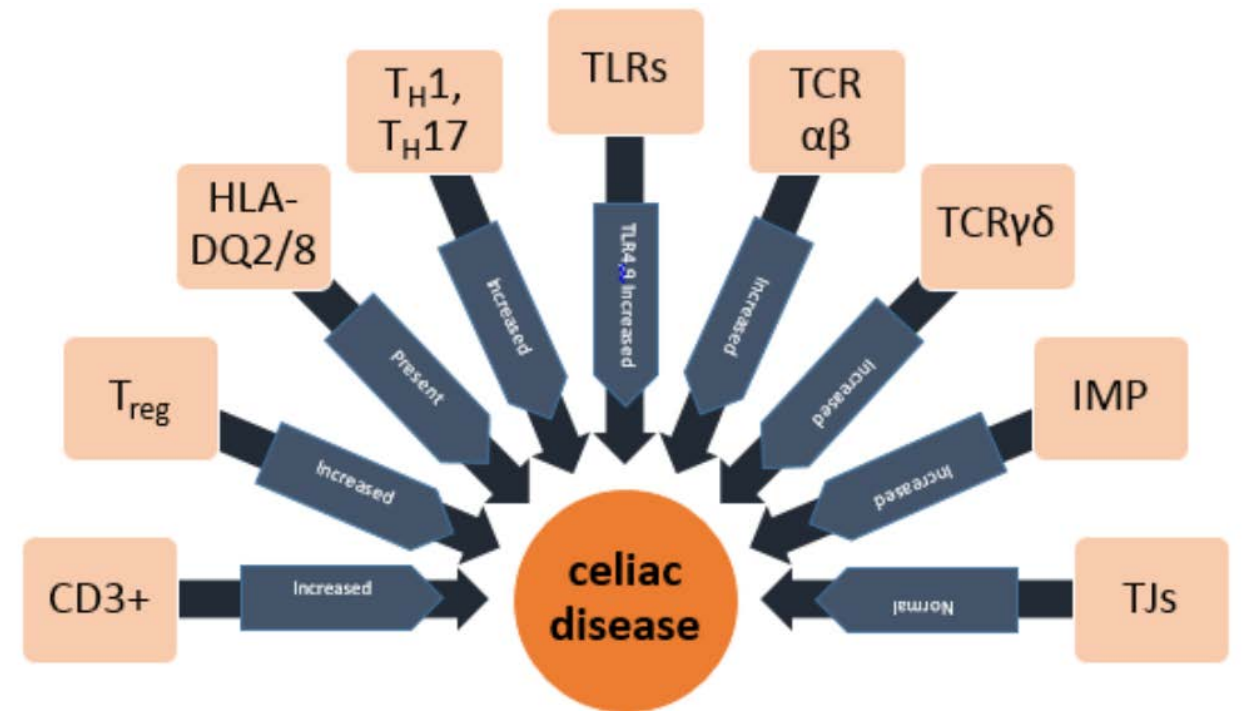

Figure 1. Immunological and intestinal epithelial barrier function findings in celiac disease 
Small intestine mucosa epithelial barrier modifications represent another possible factor involved in the pathogenesis of CD [13]. Loss of intestinal barrier function represents a key mechanism for autoimmunity development, by the continuous and aberrant passage of antigens through intestinal epithelium [14]. This may cause an immunity switch from "tolerance" to "response," hence representing an increased risk for autoimmune diseases in subjects with particular genetic determinants [13,14,15] (Figure 1).

In the intestinal epithelium, paracellular permeability is regulated by intercellular Tight junctions (TJs) and multiple proteins forming cTJs strands [16]. TJs have a critical role in the development of intestinal immunological responses. When TJs integrity is compromised, an immune response to environmental antigens, which might cross-react with host antigens, may develop, thereby triggering CD onset $[17,18]$.

Taken together these preliminary data seem to suggest that the main involvement of innate immune pathways in compare with adaptive immune pathways might help explain the clinical and serological differences in CD patients.

\section{Vaccine in Celiac Disease}

Based on our knowledge, the only treatment for CD is a lifelong GFD but in different cases GFD is not only difficult, but demanding to retain and often failed. Therefore, an effective, economical, and noninvasive alternative treatment selections for CD is need and new approaches is adjunctive and/or vaccine therapy.

New therapeutic programs could be according to the immune regulation by IL-10. In in vitro model of treated CD patients, external IL-10 can overwhelm the gliadin driven IFN- $\gamma$ response in intestinal biopsies [19]. While the high levels of IL-10 in active CD characterize a compensatory anti-inflammatory phase but is not adequate to suppress the overwhelming Th1-mediated response.

On the other hands, vaccination with gluten epitopes may prompt the extension of regulatory $\mathrm{T}$ cells, by this means refurbishing oral tolerance to gluten.

Although abundant in vivo survey have been published, however, whether these approaches can strongly decrease the inflammatory intestinal response in CD remains to be verified. A few experimental clinical trials studies have been tested and only one clinical trial has been accompanied with a gliadin-based immunotherapy (ClinicalTrials.gov Identifier: NCT00879749) [20]. This study has been completed recently and indicated a safety evaluation and estimates an inducible immune response by intradermal injections of Nexvax2 in treated CD patients which is specific to HLA-DQ2 patients.

This vaccine is contains three gluten peptides and being established by ImmunsanT for the treatment of CD. These epitopes are responsible for the various immune responses by isolated T cells [20]. This approach which a phase I in 40 HLA-DQ2+ CD patients, using subcutaneous doses showed no clinically applicable harmful phenomenon is currently in phase $1 \mathrm{~b}$ but needs confidential intradermal injections [21,22,23].

\section{Conclusion}

Current knowledge's in the pathogenesis of CD has released the entrances for a range of novel non-dietary therapy which may be used as vaccine therapy. A different approach is presently under exploration to discuss this question. So far, however, only a few numbers of experimental therapies have been investigated a new novel targets for CD in phase I-II trials and larger randomized controlled trials. The suitable unique therapy needs to be harmless, operative and inexpensive. This highlights the need for further examination into the improvement of a novel treatment possibility for CD patients.

\section{References}

[1] Rostami-Nejad M, Romanos J, Rostami K, Ganji A, EhsaniArdakani MJ, Bakhshipour AR, Zojaji H, Mohebbi SR, Zali MR, Wijmenga C. Allele and haplotype frequencies for HLA-DQ in Iranian celiac disease patients. World J Gastroenterol 2014; 20: 6302-6308.

[2] Mansueto P, Seidita A, Alcamo A, Carroccio A. Non-Celiac Gluten Sensitivity: Literature Review. J Am Coll Nutr 2014; 33:39-54.

[3] Monteleone I, Sarra M, DelVecchio Blanco G, Paoluzi OA, Franz`e E, Fina D. Characterization of IL-17A-producing cells in celiac disease mucosa. J Immunol 2010; 184: 2211-2218.

[4] Sapone A, Lammers KM, Casolaro V, Cammarota M, Giuliano MT, De Rosa M, et al. Divergence of gut permeability and mucosal immune gene expression in two gluten-associated conditions: celiac disease and gluten sensitivity. BMC Med 2011;9: 23.

[5] Sapone A, Lammers KM, Mazzarella G, Mikhailenko I, Carten`1 M, Casolaro V, Fasano A: Differential mucosal IL-17 expression in two gliadin-induced disorders: gluten sensitivity and the autoimmune enteropathy celiac disease. Int Arch Allergy Immunol 2010; 152: 75-80.

[6] Volta U, Tovoli F, Cicola R, Parisi C, FabbriA, Piscaglia M, Fiorini E, Caio G: Serological tests in gluten sensitivity (nonceliac gluten intolerance). J Clin Gastroenterol 2012;46:680-685.

[7] Le'on F, S'anchez L, Camarero C, Roy G: Cytokine production by intestinal intraepithelial lymphocyte subsets in celiac disease. Dig Dis Sci 2005.50:593-600.

[8] Fern’andez S,Molina IJ, Romero P, Gonźalez R, Pẽna J, S'anchez F, etal. Characterization of gliadin-specific Th17 cells from the mucosa of celiac disease patients. Am J Gastroenterol 2011;106:528-538.

[9] Fina D, Sarra M, Caruso R, Del Vecchio Blanco G, Pallone F, MacDonald TT, et al. Interleukin 21 contributes to the mucosal $\mathrm{T}$ helper cell type 1 response in coeliac disease. Gut 2008; 57: 887892.

[10] Eiro o N, Gonz'alez-Reyes S, Gonz'alez L, Gonz'alez LO, Altadill A, Andicoechea A, et al. Duodenal expression of toll-like receptors and interleukins are increased in both children and adult celiac patients. Dig Dis Sci 2012;57:2278-2285.

[11] Szebeni B, Veres G, Dezsofi A, Rusai K, Vannay A, Bokodi G, et al. Increased mucosal expression of toll-like receptor (TLR)2 and TLR4 in coeliac disease. J Pediatr Gastroenterol Nutr 2007;45: 187-193.

[12] Kalliom“aki M, Satokari R, L“ahteenojaH, V“ah“amiko S, Gr"onlund J, Routi T, et al. Expression of microbiota, toll-like receptors, and their regulators in the small intestinal mucosa in celiac disease. J Pediatr Gastroenterol Nutr 2012; 54:727-732.

[13] Camilleri M, Madsen K, Spiller R, Van Meerveld BG, Verne GN. Intestinal barrier function in health and gastrointestinal disease. Neurogastroenterol Motil 2012; 24:503-512.

[14] Bertolazzi S, Lanzarotto F, Zanini B, Ricci C, Villanacci V, Lanzini A. Bio-physical characteristics of gastrointestinal mucosa of celiac patients: comparison with control subjects and effect of gluten free diet. BMC Gastroenterol 2011;11:119.

[15] Visser J, Rozing J, Sapone A, Lammers K, Fasano A. Tight junctions, intestinal permeability, and autoimmunity: celiac 
disease and type 1 diabetes paradigms. Ann N Y Acad Sci 2009;1165:195-205.

[16] Drago S, El Asmar R, Di Pierro M, Grazia Clemente M, Tripathi A, Sapone A, et al. Gliadin, zonulin and gut permeability: Effects on celiac and non-celiac intestinal mucosa and intestinal cell lines. Scand J Gastroenterol 2006;41:408-419.

[17] Sander GR, Cummins AG, Henshall T, Powell BC. Rapid disruption of intestinal barrier function by gliadin involves altered expression of apical junctional proteins. FEBS Lett 2005; 579: 4851-4855.

[18] Ciccocioppo R, Finamore A, Ara C, Di Sabatino A, Mengheri E, Corazza GR. Altered expression, localization, and phosphorylation of epithelial junctional proteins in celiac disease. Am J Clin Pathol 2006;125:502-511
[19] Salvati VM, Mazzarella G, Gianfrani C, Levings MK, Stefanile R, De Giulio B, et al. Recombinant human interleukin suppresses gliadin dependent $\mathrm{T}$ cell activation in ex vivo cultured coeliac intestinal mucosa. Gut 2005; 54: 46-53.

[20] Tye-Din JA, Stewart JA, Dromey JA, Beissbarth T, van Heel DA Tatham A, et al. Comprehensive, quantitative mapping of $\mathrm{T}$ cell epitopes in gluten in celiac disease. Sci Transl Med 2010; 2: 41-51.

[21] Stein J, Schuppan D. Coeliac Disease - New Pathophysiological Findings and Their Implications for Therapy. Viszeralmedizin. 2014;30:156-165.

[22] Riedmann EM. Human vaccines: news. Hum Vaccin Immunother. 2012; 8:1550-1553.

[23] Crespo Pérez L, Castillejo de Villasante G, Cano Ruiz A, León F. Non-dietary therapeutic clinical trials in coeliac disease. Eur J Inter Med. 2012; 23: 9-14. 